\title{
How Microbiome Impact on the Cardiovascular System
}

\author{
Constantin V Sobol*
}

Sechenov Institute of Evolutionary Physiology and Biochemistry, Russian Academy of Science, Russia

Received: November 20, 2013; Accepted: March 08, 2014, Published: March 12, 2014

*Corresponding author: Constantin V Sobol, Sechenov Institute of Evolutionary Physiology and Biochemistry, Russian Academy of Science, Thorez 44 St, Petersburg, Russia, Fax: 7812 552-1180; E-mail: sobol_cv@yahoo.com

\begin{abstract}
Humans are known to have constant symbiosis with microorganisms. Non-pathogenic microbial associations participate in vital functions of a host macroorganism and in maintenance of homeostasis. There is some data that our microflora may negatively influence on host macroorganism. Gut flora metabolism of phosphatidylcholine may promote cardiovascular disease [1,2]. However, Lactobacilli and products of their metabolism, being administered with a food, have positive impact on the cardiovascular system. Bioactive peptides produced during Lactobacillus fermentation are favorable for the treatment of hypertension. Probiotic bacteria have demonstrated the ability to reduce blood cholesterol by several mechanisms including assimilation of cholesterol. Metabolic products of lactobacilli may stimulate intracellular calcium signaling in cells of cardiovascular system. We should use complete potential of proteolytic activity of Lactobacilli, their ability to produce high concentration of favorable metabolites to receive unique pharmabiotic products for prophylaxis and probably for the treatment of life-threatening diseases.
\end{abstract}

Keywords: Lactobacillus fermentation; Probiotics; Microbiome; Cardiovascular system; Vascular smooth muscle; Heart; Hypertension; Cholesterol

\section{Abbreviation}

ACE: Angiotensin-converting enzyme; CVD: Cardiovascular disease; SHR: Spontaneously hypertensive rats; TMAO: Trimethylamine-N-oxide

Humans are known to have constant symbiosis with microorganisms (microbiota/microflora). Non-pathogenic microbial associations participate in vital functions of a host macroorganism and in maintenance of homeostasis. Actually, microbiota may be considered as an endocrine organ of a host macroorganism[3]. It has morphokinetic influence, participate in a metabolic activity and produce biologically active compounds, including important neuromediators regulating mental activity of human, such as glutamate and $\gamma$-aminobutyric acid [3].

The indigenous microflora including intestinal one, colonizing the sites of macroorganism and connecting to the external environment, is separated from the internal environment of a macroorganism by corresponding barriers. However, metabolic products of our microflora in gastrointestinal tract can rather easily penetrate into the blood, thereby influencing the macroorganism physiology.

There are some data that our microflora may negatively influence on host macroorganism. There is some evidence of microbe-induced human malignancies [4]. There is some interconnection of the gut violation and cardiac diseases[5]. Recently it was shown that gut flora metabolism of phosphatidylcholine promotes cardiovascular disease. An increased plasma level of trimethylamine-N-oxide (TMAO), a product of the intestinal microbial metabolism of dietary phosphatidylcholine is associated with an increased rate of major adverse cardiovascular events. A major adverse cardiovascular event was defined as death, myocardial infarction, or stroke. Actually, fasting plasma TMAO levels predict the risk of incident major adverse cardiovascular events independently of traditional cardiovascular risk factors [1,2].However, there is no the mechanistic link between TMAO and cardiovascular risk, because of an efficient kidney excretion mechanism [2]. An association between infectious organisms and atherosclerosis has previously been postulated [6]. However anti-microbial therapy in preventing disease progression has been disappointing [6,7].In their latest study, published in the New England Journal of Medicine,[2] suggested therapeutic modulation of microbiota with functional food containing probiotics [8] and with nonsystemic antibiotics reduction of TMAO producing microbes. However, potency of probiotic to reduce TMAO is strain dependent [8]. Additionally, macroorganism may have non-toxic bacteria involved in its microflora, which are able to reduce TMAO. For example, E-coli TMAO reduction was associated with an anaerobic respiration [9].

Authors [2] recommend avoiding excessive consumption of dietary phosphatidylcholine and choline. Choline intake can be reduced with a vegetarian or high-fibre diet, but choline is a semiessential nutrient, so should not be entirely eliminated from the diet [2].

I think that consumption of non-fat food fermented with Lactobacilli may be effectively used for prophylaxis of cardiovascular disease. Lactobacilli and products of their metabolism, being administered with a food, have positive impact on the cardiovascular system [10]. Lactobacilli are able to produce biologically active peptides inhibiting Angiotensin- 
Converting Enzyme (ACE). Hypertension arises from the conversion of Angiotensin-I to the potent vasoconstrictor Angiotensin-II by the ACE. Dairy products fermented with Lactobacilli contain bioactive peptides known to inhibit the activity of ACE and thus alleviate hypertension. Oral administration of Calpis sour milk to spontaneously hypertensive rats (SHR) was able to lower systolic blood pressure [11]. Similar results were seen with oral administration of milk fermented with L. helveticus CP790 [12].Studies in humans have shown that in persons who fed milk fermented with Lactobacilli a significant lowering of blood pressure was observed $[13,14]$. Thus, bioactive peptides produced during Lactobacillus fermentation are favorable for the treatment of hypertension. The ACE-inhibitory peptides produced during the fermentation of milk are already the basis for health claims associated with some fermented milk products on the market, such as Calpis (Calpis Co., Ltd., Japan) and Evolus ${ }^{\circledR}$ (Valio, Finland) [15].

Hypertension is often associated with hypercholesterolemia or lipid abnormality and obesity [16]. Hypercholesterolemia is another significant risk factor for cardiovascular disease. Cholesterol is depositing in the arteries restricting blood flow to the heart. Probiotic bacteria have demonstrated the ability to reduce blood cholesterol by several mechanisms including assimilation of cholesterol [17],binding cholesterol and bile acids to the cell surface thus inhibiting absorption from the small intestine [18],and suppression of bile acid absorption by deconjugation of bile salts by the bacterial bile salt hydrolase activity $[19,20]$ and may reduce oxidized low-density lipoproteins [21],thereby reducing cardiovascular complications. Cholesterol-lowering effect of probiotics was observed in hyper-cholesterolemic hamsters [22] and in rat [23].In humans, hypercholesterolemic patients fed probiotics yogurt containing L. acidophilus and B. lactis were able to reduce their cholesterol levels compared to cohorts who consumed ordinary yogurt [24, 25].Nevertheless, some authors did not observe cholesterollowering effect of probiotics [15].

At last, administration of Probiotics and products of their metabolism improves gastrointestinal barrier, thereby reducing complications in patients with chronic heart insufficiency [5]. Administration of Probiotics in intensive care units improved the immunity state, decreased overall mortality, and reduced such symptoms as diarrhea and sepsis [26].

At the cellular level it was shown that some products of a bacterial fermentation and organic acids, such as lactate, acetate, propionate, and butyrate caused contraction of colon smooth muscle via calcium influx into the cells [27]. It was shown that a bacterial supernatant E. coli Nissle 1917 increases colon contraction via non-identified metabolites in addition to acetate [28].However, there is the data showing that acetate, propionate and butyrate do not increase intracellular calcium in colon smooth muscles [29].Recently, it was shown that Lactobacilli are able to increase intracellular calcium in cardiomyocytes, thereby increasing contractility of myocardium [30], in contrast to bacterial DNA and RNA of pathogenic microorganisms ( $S$. aureusand E. coli) and LPS, which suppressed contractility of rat cardiomyocytes significantly [31]. In neurons probiotic metabolic products stimulated protein kinase-C activity and partly activated ryanodine receptors [32].In vascular smooth muscles probiotic metabolic products also stimulated protein kinase C [33], stimulated heart mitochondria respiration and exerted a mild uncoupling effect on electronic transport and oxidative phosphorylation in mitochondria [30].

Earlier it was shown that intravenous administration of the cultural milieu of lactic bacteria was able to protect myocardium from ischemia [34]. From the data provided it is clear that components of the cultural solution of Lactobacillus bulgaricus-51 cause hypotension, poorly influencing heart beating [34]. These data are in accordance with our experiments, in which lactobacilli can suppress considerably the thapsigargininduced calcium influx in vascular smooth muscle cells [30]. In the conditions of sepsis, steady hypotension is observed and the agonist-induced calcium influx is considerably suppressed, which worsen the situation. Endotoxins also inhibit both phases of the calcium response considerably, namely, calcium release from intracellular stores and, to a great extent, agonist-induced calcium influx in endothelial cells of blood vessels [35]. Thus, during sepsis, when considerable bacterial translocation is observed, a large amount of lactic bacteria in the blood may aggravate a septic disease by increasing hypotension.

Despite the fact that lactic bacteria practically do not cause infectious diseases, nevertheless, there are some data where Lactobacilliare associated with cardiovascular infections [36]. Moreover, it was shown that after single intraperitoneal injection of the Lactobacillus caseicellular extract in mice, the inflammation of coronary arteries, similar to the Kawasaki disease, is developed [37]. In Europe, North America, and Japan the Kawasaki disease is considered to be the main reason of the acquired cardiovascular sickness in children [38].

However, various fermentative products of Lactobacilli have long save history of administration for thousands of years, in the form of fermented milk and vegetable products such as yogurt and pickles [39]. Such low fat fermented products may be used for the prophylaxis of some cardiovascular diseases, mentioned above. The problem is that yogurts at modern market generally contain high amount of carbohydrates. Consumption of the latter has been shown to increase the risk to develop CVD [40]. Thus, positive effect of fermented probiotic products may be annihilated, if not more, by negative effect of carbohydrates.

Fermentation of food products is very promising method of receiving biologically active natural products, which have positive impact on the cardiovascular system. Such products contain metabolites of proteolytic activity of fermenting bacteria. There are huge fermentative products: Bulgarian yogurt, kefir, koumiss, Italian cheeses, Swedish and Finnish fermented milk, Russian ryazhenka and prostokvasha, Sour Cream, French Creme fraiche, etc. National food of Japan, New Zealand, Africa (cassava) and many othersmost fermented food products, such as yogurt, fermented beverages or juices and cheese, are taken only through the first stage of fermentation, intended simply to pre-digest or 
preserve the food (with organic acids, etc. produced by bacteria) while most organic matter remains in complex form. We should use complete potential of proteolytic activity of Lactobacilli, their ability to produce high concentration of favorable metabolites to receive unique pharmabiotic products for prophylaxis and, probably, for the treatment of life-threatening diseases.

\section{References}

1. Wang Z, Klipfell E, Bennett BJ, Koeth R, Bruce S, et al. (2011) Gut flora metabolism of phosphatidylcholine promotes cardiovascular disease. Nature 472: 57-63.

2. Tang WH, Wang Z, Levison BS, Koeth RA, Bruce S Levison, et al. (2013) Intestinal Microbial Metabolism of Phosphatidylcholine and Cardiovascular Risk. N Engl J Med 368: 1575-1584.

3. Evans JM, Morris LS, Marchesi JR (2013) The gut microbiome: the role of a virtual organ in the endocrinology of the host. J Endocrinol 218(3): R37-47.

4. Plottel CS, Blaser MJ (2011) Microbiome and Malignancy. Cell Host \& Microbe 10(4): 324-335.

5. Krack A, Sharma R, Figulla HR, Anker SD (2005) The importance of the gastrointestinal system in the pathogenesis of heart failure. Eur Heart J 26(22): 2368-2374.

6. Shah PK (2001) Link between infection and atherosclerosis: who are the culprits: viruses, bacteria, both, or neither? Circulation 103: 5-6.

7. Grayston JT, Kronmal RA, Jackson LA, Parisi AF, Muhlestein JB, et al. (2005) Azithromycin for the secondary prevention of coronary events. N Engl J Med 352(16): 1637-1645.

8. Martin FPJ, Wang Y, Sprenger N, Yap IKS, Lundstedt T, et al. (2008) Probiotic modulation of symbiotic gut microbial-host metabolic interactions in a humanized microbiome mouse model. Mol Syst Biol 4: 157 .

9. Barrett EL, Kwan HS (1985) Bacterial reduction of trimethylamine oxide. Ann Rev Microbiol 39: 131-149.

10. Sonestedt E, Wirfalt E, Wallstrom P, Gullberg B, Orho MM, et al. (2011) Dairy products and its association with incidence of cardiovascular disease: the Malmo diet and cancer cohort. Eur J Epidemiol 26(8): 609-618.

11. Nakamura Y, Yamamoto N, Sakai K, Takano T (1995) Antihypertensive effect of sour milk and peptides isolated from it that are inhibitors of Angiotensin-1 converting enzyme. J Dairy Sci 78(6): 1253-1257.

12. Yamamoto N, Akino A, Takano T (1994) Antihypertensive effects of the peptides derived from casein by an extracellular proteinase from Lactobacillus helveticus CP970. J Dairy Sci 77(4): 917-922.

13. Seppo L, Jauhiainen T, Poussa T, Korpela R (2003) A fermented milk high in bioactive peptides has a blood pressure-lowering effect in hypertensive subjects. Am J Clin Nutr 77(2): 326-330.

14. Aihara K, Kajimoto O, Hirata H, Takahashi R, Nakamura Y (2005) Effect of powdered fermented milk with Lactobacillus helveticus on subjects with high-normal blood pressure of mild hypertension. J Am Coll Nutr 24(4): 257-265.

15. Liong MT (2007) Probiotics: A Critical Review of Their Potential Role as Antihypertensives, Immune Modulators, Hypocholesterolemics, and Perimenopausal Treatments. Nutr Rev 65(7): 316-328.

16. Yekeen LA, Sanusi RA, Ketiku AO (2003) Prevalence of obesity and high level of cholesterol in hypertension: Analysis of data from the university college hospital, Ibadan. Afr J Biomed Res 6: 129-132.

17. Gilliland SE, Nelson CR, Maxwell C (1985) Assimilation of cholesterol by Lactobacillus acidophilus. App Environ Microbiol 49(2): 377-381.

18. Danielson AD, Peo ER, Shahani KM, Lewis AJ, Whalen PJ, et al. (1989) Anticholesterolemic property of Lactobacillus acidophilus yoghurt fed to mature boars. J Anim Sci 67: 966-974.

19. De Smet I, De Boever P, Versteaete W (1998) Cholesterol lowering in pigs through enhanced bacterial bile salt hydrolase activity. British J Nutr 79(2): 185-194.

20. Liong MT, Shah NP, (2005). Bile salt deconjugation ability, bile salt hydrolase activity and cholesterol co-precipitation ability of lactobacilli strains. Int Dairy J, 15, 391-398. Missed in printed manuscript.

21. Mikelsaar M, Stsepetova J, Hutt P, Kolk H, Loivukene K, et al. (2010) Intestinal Lactobacillus sp. is associated with some cellular and metabolic characteristics of blood in elderly people. Anaerobe 16(3): 240-246.

22. Bhathena J, Martoni C, Kulamarva A, Urbanska AM, Malhotra M, et al. (2009) Orally delivered microencapsulated live probiotic formulation lowers serum lipids in hypercholesterolemic hamsters. J Med Food 12(2): 309-310.

23. Wang Y, Xu N, Xi A, Ahmed Z, Bai X, et al. (2009) Effects of Lactobacillus plantarum MA2 isolated from Tibet kefir on lipid metabolism and intestinal microflora of rats fed on high-cholesterol diet. Appl Microbiol Biotechnol 84(2): 341-347.

24. Ataie-Jafari A, Larijani B, Alavi Majd H, Tahbaz F (2009) Cholesterollowering effect of probiotic yogurt in comparison with ordinary yogurt in mildly to moderately hypercholesterolemic subjects. Ann Nutr Metabol 54(1): 22-27.

25.Ejtahed HS, Mohtadi-Nia J, Homayouni-Rad A, Niafar M, Asghari JM, et al. (2011) Effect of probiotic yogurt containing Lactobacillus acidophilus and Bifidobacterium lactis on lipid profile in individuals with type 2 diabetes mellitus. J Dairy Sci 94: 3288-3294.

26. Madsen K (2008) Probiotics in critically ill patients. J Clin Gastroenterol 42: S116-S118.

27. Rondeau MP, Meltzer K, Michel KE, McManus CM, Washabau RJ (2003) Short chain fatty acids stimulate feline colonic smooth muscle contraction. J Feline Med Surgery 5(3): 167-173.

28. Bar F, Von KH, Roblick U, Bruch HP, Schulze L, et al. (2009) Cell free supernatants of Escherichia coli Nissle 1917 modulate human colonic motility: Evidence from an in vitro organ bath study. Neurogastroenterol Motil 21(5): 559-566.

29. Abrahamse SL, Pool-Zobel BL, Rechkemmer G (1999) Potential of short chain fatty acids to modulate the induction of DNA damage and changes in the intracellular calcium concentration by oxidative stress in isolated rat distal colon cells. Carcinogenesis 20(4): 629-634.

30.Sobol CV, Korotkov SM, Belostotskaya GB, Nesterov VP (2013) The Influence of Probiotics and Probiotic Product on Respiration of Mitochondria and Intracellular Calcium Signal in Cells of Cardiovascular System. Biochem (Moscow) Suppl Ser A: Membrane and Cell Biology 7(4): 294-301.

31. Paladugu B, Kumar A, Parrillo JE, Der S, Osman J, et al. (2004) Bacterial DNA and RNA induce rat cardiac myocyte contraction depression in vitro. Shock 21(4): 364-369.

32. Sobol CV, Belostotskaya GB, (2005). Ca2+ signal in rat brain neurons upon application of probiotic product. J Neurochem, 94 (Suppl.1), 85. 
33. Sobol CV (1995) Mechanisms of vasoconstriction induced in frog vascular smooth muscle by MD1, a new biotechnological agent. Gen Physiol Biophys 14(4): 293-303.

34. Oxman T, Shapira M, Diver A, Klein R, Avazov N, et al. (2000) A new method of long-term preventive cardioprotection using Lactobacillus. Am J Physiol Heart Circ Physiol 278(5): H1717-H1724.

35. Graier WF, Myers PR, Rubin LJ, Adams HR, Parker JL (1994) Escherichia coli endotoxin inhibits agonist mediated cytosolic $\mathrm{Ca}^{2+}$ mobilization and nitric oxide biosynthesis in cultured endothelial cells. Circ Res 75(4): 659-668.

36. Yagi S, Akaike M, Fujimura M, Ise T, Yoshida S, et al. (2008) Infective endocarditis caused by lactobacillus. Intern Med 47(12): 1113-1116.

37. Rosenkranz ME, Schulte DJ, Agle LMA, Wong MH, Zhang W, et al.
(2005) TLR2 and MyD88 Contribute to Lactobacillus casei ExtractInduced Focal Coronary Arthritis in a Mouse Model of Kawasaki Disease. Circulation 112(19): 2966-2973.

38. Bronstein DE, Dille AN, Austin JR, Williams CM, Palinkas LA, et al. (2000) Relationship of climate ethnicity and socioeconomic status of Kawasaki disease in San Diego County, 1994 through 1998. Pediatr Infect Dis J 19(11): 1087-1091.

39. Mateos JA (2004) Yoghurt: a probiotic Mediterranean food. Arch Latinoam Nutr 54: 76-78.

40. World Health Organization (2003) Diet, nutrition and the prevention of chronic diseases: report of a joint WHO/FAO expert consultation 916. 